\title{
Mycobacterium ulcerans Disease with Unusual Sites Not to Be Ignored
}

\author{
Sangaré Abdoulaye, Kourouma Sarah Hamdan, Kouassi Yao Isidore, Ecra Elidjé Joseph, \\ Kaloga Mamadou, and Gbery Ildevert Patrice
}

Department of Dermatology of the University Teaching Hospital of Treichville, Riviera, BP 408 Cidex 03 Abidjan, Cote d'Ivoire

Correspondence should be addressed to Sangaré Abdoulaye; sang_abdoulaye@yahoo.fr

Received 17 June 2014; Revised 12 September 2014; Accepted 14 September 2014; Published 16 October 2014

Academic Editor: H. Honigsmann

Copyright (c) 2014 Sangaré Abdoulaye et al. This is an open access article distributed under the Creative Commons Attribution License, which permits unrestricted use, distribution, and reproduction in any medium, provided the original work is properly cited.

Objective. The usual preferential site of BU is in the limbs. In our experience, we noticed atypical and often misleading sites which pose serious issues for the diagnosis and often for the treatment. Methods. This is a retrospective study conducted over a period of ten years of BU treatment at the Department of Dermatology of the University Teaching Hospital of Treichville (Abidjan, Côte d'Ivoire). We included in this study all BU cases with atypical site diagnosed clinically and confirmed either by the histology, by smear, or by PCR. Results. Epidemiologically, the age of patients ranged from 3 to 72 years with a median age of 14.2 years. Children aged less than 15 years were affected in almost $80 \%$ of case. The clinical table was dominated by ulcerated forms in $82.1 \%$ of cases. The unusual topography mostly observed was that of the torso (thorax, back, and abdomen) in $76.8 \%$ of cases. Conclusion. BU is an endemic disease in Côte d'Ivoire where it constitutes a serious public health issue. Several years following its first cases, BU still is little known. This dermatosis may present atypical misleading clinical aspects which must be ignored.

\section{Introduction}

Mycobacterium ulcerans (MU) also known as Buruli ulcer (BU) named after the District of Uganda where an epidemic occurred in the 1960s is mycobacteriosis [1]. This disease believed to be mysterious by many parents is characterized by preulcerative lesions leading in the long term to major chronic cutaneous deterioration often associated to definitive disabilities [2]. In Côte d'Ivoire, Buruli ulcer which is the second mycobacteriosis after tuberculosis constitutes an emerging endemic. This is the reason why the government initiated, since 1998, the National Programme of Fight against Mycobacterium Ulcers (PNUM) in Côte d'Ivoire.

Its preferential site in 9 out of 10 cases is in lower limbs $[3,4]$. However, in our experience, we observed some unusual sites. So the purpose of this study is to contribute to a better understanding of them.

The specific objectives of this study are to determine sociodemographic characteristics and to describe clinical and topographical aspects of such unusual sites.

\section{Patients and Method}

This is a retrospective, cross-sectional, and descriptive study related to BU cases observed over a period of ten years (i.e., from 2003 to 2013). This study was conducted in the Dermatology Department of the University Teaching Hospital of Treichville which is the reference centre for cutaneous pathologies in Abidjan and served as the head office of the PNUM.

We included patients, irrespective of their gender and age, who over the study period developed an unusual (atypical) ulcer or a nodule clinically evoking Mycobacterium ulcerans.

We considered, as the usual or typical site of BU, any ulcer that is found on the limbs and more specifically on lower limbs.

However, any site, other than the limb, is said to be unusual, atypical, or misleading. The subject matter of this study is unusual sites.

The BU was diagnosed on the basis of clinical and paraclinical arguments. 
With regard to clinical aspects, we considered the existence of the following:

(i) manifestations which evoke the inception of a BU: nodule, oedema, and infiltrated plate,

(ii) at latter stage, the characteristic ulceration with its thickened, devitalized, and peeled edges, surpassing the base.

With regard to paraclinical aspects, there should be at least the result of one of the following examinations:

(i) the histology of a nodule, an oedema, or an infiltrated plate with Ziehl-Neelsen stain;

(ii) the smear conducted from the exudates of the ulceration edges with Ziehl-Neelsen stain;

(iii) the PCR (polymerase chain reaction) conducted on the exudate.

Cutaneous biopsies were conducted at the Department of Dermatology and plates were read in the anatomic pathology laboratory of the same University Teaching Hospital. The smear and PCR were conducted by the "Institut Pasteur of Côte d'Ivoire."

The histology was revelatory of a BU case if an infiltrate of lymphocyte, histiocytosis, and hypodermic necrosis were found or if AFB (acid-alcohol-fast Bacilli) were revealed by the Ziehl-Neelsen stain method.

With regard to smear, a positive Ziehl-Neelsen stain was considered as a potential BU case. However, when the ZiehlNeelsen stain was negative, a PCR (polymerase chain reaction) was conducted on the sampling in order to confirm the diagnosis.

The smear and histology are less expensive but they have an average sensibility. Moreover, such examinations have a poor specificity and do not permit discriminating mycobacteria.

With regard to PCR, its sensibility and specificity are above $90 \%$.

On the basis of clinical and paraclinical arguments, we collected in all $213 \mathrm{BU}$ records comprising classic sites as well as unusual sites.

We did not include in this study all the incomplete records which had no paraclinical data.

\section{Results}

3.1. Overall Incidence of BU during the Study Period. During the study period, we recorded in the whole department 42495 patients who came for consultation for various dermatosis. Of the whole population who came for consultation in our department over the study period, we observed 213 BU cases, that is, an overall incidence of $0.5 \%$.

\subsection{Sociodemographic Characteristics of Atypical BU (Table 1)}

3.2.1. Incidence of $B U$ with Atypical Site. Of the 213 cases of BU collected, we observed 39 cases of BU with atypical site (i.e., $18.3 \%)$ and 174 cases of BU found on the limbs (81.6\%).
TABLE 1: Epidemiological and clinical characteristics of atypical forms.

\begin{tabular}{|c|c|c|}
\hline Parameters & $\begin{array}{c}\text { Numbers } \\
(N)\end{array}$ & $\begin{array}{c}\text { Percentage } \\
\text { (\%) }\end{array}$ \\
\hline \multicolumn{3}{|c|}{ Incidences of atypical site } \\
\hline Sites on the limbs & 174 & 81.6 \\
\hline Atypical sites & 39 & 18.3 \\
\hline \multicolumn{3}{|c|}{ Sociodemographic characteristics } \\
\hline \multicolumn{3}{|l|}{ Age } \\
\hline$<$ or $=15$ years & 31 & 79.5 \\
\hline$>15$ & 08 & 20.5 \\
\hline \multicolumn{3}{|l|}{ Sex } \\
\hline Female & 28 & 71.7 \\
\hline Male & 11 & 28.3 \\
\hline \multicolumn{3}{|l|}{ Residence } \\
\hline Swampy zones & 30 & 77 \\
\hline Far & 09 & 23 \\
\hline \multicolumn{3}{|l|}{ Clinical aspects } \\
\hline Ulcerated forms & 32 & 82.1 \\
\hline Edematous forms & 04 & 10.2 \\
\hline Nodular forms & 03 & 7.7 \\
\hline \multicolumn{3}{|l|}{ Topographic aspects } \\
\hline Thorax & 12 & 30.7 \\
\hline Abdomen & 10 & 25.6 \\
\hline Back & 08 & 20.5 \\
\hline Face & 06 & 15.3 \\
\hline Genitals & 03 & 7.7 \\
\hline
\end{tabular}

3.2.2. Age of Patients with BU of Atypical Site. The age of patients ranged from 3 to 72 years. The mean age was 14.2 years. Children aged less than 15 years were affected in almost $80 \%$ of the cases.

3.2.3. Gender of Patients with BU of Atypical Site. We observed a female predominance of $71.7 \%$. The sex ratio was 2.5.

3.3. Clinical Characteristics of BU Cases with Atypical Site. Clinical forms of atypical site were dominated by ulcerated forms $(82.1 \%)$.

3.4. Topographical Aspects of BU with Atypical Site. Sites on the torso (thorax, abdomen, and back) were the most frequent forms $(76.8 \%)$.

\section{Discussion}

$\mathrm{BU}$ is a mycobacteriosis which rages under the form of endemic foci in our country to the extent that, in 1995, the Ivorian government set up a National Programme of Fight against Mycobacterium Ulcers (PNUM). Unlike its usual sites in the limbs which are well documented, atypical sites are not. As a matter of fact, they are misleading forms 
whose diagnosis and treatment are difficult and should not be ignored by practitioners; they are likely to threaten the functional prognosis and survival in some cases. Such forms in our study had a hospital incidence of $18.3 \%$.

Sociodemographic characteristics of misleading forms are similar to usual forms of BU. BU with atypical sites affects, like its classic form, mostly children. In $79.5 \%$ of the cases, atypical forms were observed in children aged less than 15 years. The BU predominance in this target is observed in various studies [5-7]. It was related to a deficit of immunity in those children [8]. The factor accounting for that situation is the absence of specific vaccine protection against $\mathrm{MU}$ and the antituberculosis vaccination, BCG (Bacilli CalmetteGuerin), offers only a transitional protection which subsides from 6 months to 1 year $[9,10]$. Moreover, games or fishing, by those children near waters, exposes them to cutaneous microtrauma which favours the penetration of $\mathrm{MU}$ in the body [11].

In our study, females patients were the most affected people. The epidemiological profile classically shows that the BU affects the children without distinction of sex. This ascendancy of females in this study would be of recruitment bias.

They represent $71.7 \%$ of patients. As a matter of fact, women, in our traditions, are in charge of household chores which are mainly laundry and dishes. These chores are also conducted near stretch of water and swamps in $77 \%$ of the cases (please refer to Table 1).

Our country, Côte d'Ivoire, is a country with limited resources. The minimum wage is $\$ 120$. Due to poverty, only few households have access to drinking water. As a result, many families are obliged to use swamp water for the needs of their household. Though the BU transmission mode is not clearly identified, one knows that contact with those stagnant waters is a major factor in the outbreak of the disease [12]. As a matter of fact, a PCR conducted enabled us to discover freshwater bugs of the like of Naucoris and Diplonychus on the roots of some aquatic plants which might shelter MU [1].

In our experience, patients are barely consulted at the inception which is oedema (10.2\%) and nodule (7.7\%). However, when the disease is diagnosed at this stage, the treatment is less complex and the prognosis is better [13]. However, in $82.1 \%$ of the cases, patients go to hospital at the ulcerative stage which is the severest form, the most dilapidating, with at times a risk of incapacitating scares in children [14]. This negligence of diseases can be explained by poverty. As a matter of fact, due to economic reasons, those patients undertake self-medication at the inception of the pathology. They would only go to health centres, after several weeks or months when their treatments have failed or when the case has developed into some complications. As well, those unusual sites of BU are sometimes very misleading and give rise to misdiagnosis and delays in the efficient treatment, given that it is ignored by many practitioners. This is the reason of our vehement advice to our colleagues in endemic zones to undertake in case of doubt the incisional biopsy of any nodule in order to conduct histological examination and, at the ulceration stage, conduct wound edge swabbing in view

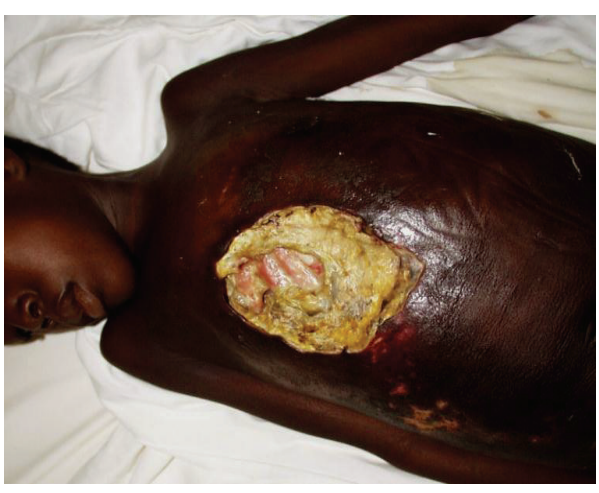

FIgURE 1: Thoracic BU revealing the ribs of an 8-year-old girl.

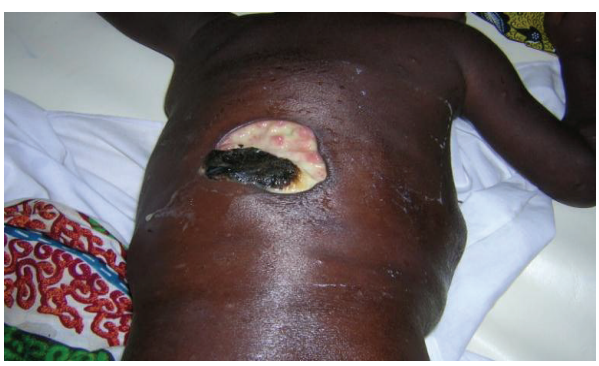

FIGURE 2: BU of the back.

of conducting a PCR which would allow for early diagnosis of BU within 48 hours $[15,16]$.

Histology and smear are examinations with an average sensibility and a poor specificity. The poor performance of these examinations could actually induce a bias of recruitment by omitting confirmed cases of $\mathrm{BU}$ or registering false cases. However given that these tests are less expensive and easy to carry out, they permit defining probable cases of $\mathrm{BU}$ in endemic zones like ours, according to the WHO [17].

However, the PCR has quite a good sensibility and its specificity is above $90 \%$. In the event of a positive result, it allows the confirmation of BU cases [17]. But its high cost prevents its use as a routine examination.

With regard to topography, BU may affect any part of the human body but limbs remain its predilection site [18-20]. Unusual topographic aspects observed were predominantly in the torso (thorax, abdomen, and back) in $76.8 \%$ (Figures 1 and 2). There are severe forms which could threaten survival due to pneumothorax type complications or pleurisy which go along with them in some cases [21].

Apart from these predominant forms found on the torso, the study revealed moreover facial affections of up to $15.3 \%$ (Figure 3). These forms located on the face, in addition to presenting diagnostic difficulties, pose a problem with regard to their surgical treatment given their proximity to the eyes. The functional prognostic of these forms is related to the likeliness of extension of BU to the eyes as it was observed with some patients. 


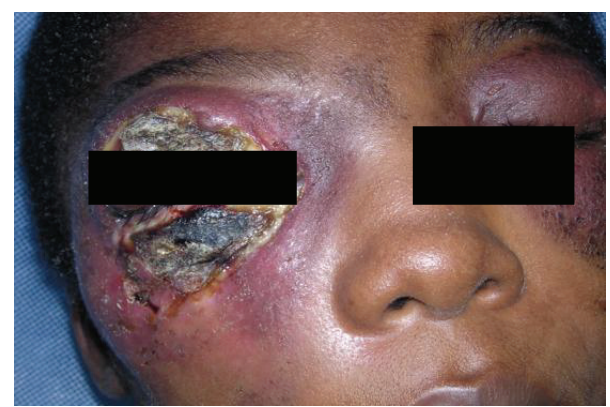

FIgURE 3: Facial BU above and beneath right palpebral fissure.

\section{Conclusion}

BU is an endemic disease in Côte d'Ivoire where it constitutes a serious public health issue. Several years following its first description by Mac Callum, BU remains understudied. As a matter of fact, in addition to its mode of transmission which is yet to be elucidated, this dermatosis may clinically present atypical and misleading aspects likely to threaten survival. Future researches could help for a better understanding of the various unknown aspects of this disease.

\section{Abbreviations}

AFB: Acid-alcohol-fast Bacilli

BCG: Bacilli Calmette-Guerin

MU: $\quad$ Mycobacterium ulcerans

PCR: Polymerase chain reaction

PNUM: National Programme of Fight against Mycobacterium Ulcers

B.U: $\quad$ Buruli ulcers.

\section{Conflict of Interests}

The authors declare that there is no conflict of interests regarding the publication of this paper.

\section{References}

[1] J. M. Kanga, E. D. Kacou, K. Kouame et al., "Buruli ulcer: epidemiological, clinical and therapeutic aspects in Côte d'Ivoire," Medecine Tropicale, vol. 64, no. 3, pp. 238-242, 2004.

[2] R. Josse, A. Guedenon, H. Darie, S. Anagonou, F. Portaels, and W. M. Meyers, "Mycobacterium ulcerans skin infection: Buruli ulcers," Medecine Tropicale, vol. 55, no. 4, pp. 363-373, 1995.

[3] L. S. B. Manou, F. Portaels, M. Eddyani, A. U. Book, K. Vandelannoote, and B. C. de Jong, "Mycobacterium ulcerans disease (Buruli ulcer) in Gabon: 2005-2011," Medecine et Sante Tropicales, vol. 23, no. 4, pp. 450-457, 2013.

[4] K. Kollie, Y. A. Amoako, J. Ake et al., "Buruli ulcer in Liberia, 2012," Emerging Infectious Diseases, vol. 20, no. 3, pp. 494-496, 2014.

[5] B. Saka, D. E. Landoh, B. Kobara et al., "Profile of Buruli ulcer treated at the national reference centre of Togo: a study of 119 cases," Bulletin de la Societe de Pathologie Exotique, vol. 106, no. 1, pp. 32-36, 2013.
[6] J. M. Kanga, D. E. Kacou, A. Sangaré, Y. Dabila, N. H. Asse, and S. Djakeaux, "Recurrence cases observed after surgical treatment of Buruli ulcer in Cote d'Ivoire," Bulletin de la Societe de Pathologie Exotique, vol. 96, no. 5, pp. 406-409, 2004.

[7] H. Darie, "Mycobacterium ulcerans infection: epidemiological, clinical and therapeutical aspects," Bulletin de la Societe de Pathologie Exotique, vol. 96, no. 5, pp. 368-371, 2004.

[8] J. M. Kanga, E. D. Kacou, K. Kouamé et al., "Fighting against Buruli ulcer: the Côte-d'Ivoire experience," Bulletin de la Societe de Pathologie Exotique, vol. 99, no. 1, pp. 34-38, 2006.

[9] A. Sangaré, E. Ecra, M. Kouyaté, C. Ahogo, and M. Kaloga, "Epidemiological profile of Buruli Ulcer in Abidjan," Afrique Biomédicale, vol. 11, no. 2, pp. 20-25, 2006.

[10] P. J. Converse, D. V. Almeida, E. L. Nuermberger, and J. H. Grosset, "BCG-mediated protection against Mycobacterium ulcerans infection in the mouse," PLoS Neglected Tropical Diseases, vol. 5, no. 3, article e985, 2011.

[11] M. W. Bratschi, M. T. Ruf, A. Andreoli et al., "Mycobacterium ulcerans persistence at a village water source of Buruli ulcer patients," PLoS Neglected Tropical Diseases, vol. 8, no. 3, Article ID e2756, 2014.

[12] K. H. Jacobsen and J. J. Padgett, "Risk factors for Mycobacterium ulcerans infection," International Journal of Infectious Diseases, vol. 14, no. 8, pp. e677-e681, 2010.

[13] E. Ecra, J. M. Kanga, I. D. Gbery et al., "Detection and treatment of early forms of Mycobacterium ulcerans infection in Ivory Coast," Médecine tropicale : revue du Corps de santé colonial., vol. 65, no. 4, pp. 334-338, 2005.

[14] T. S. van Der Werf, W. T. A. van Der Graaf, J. W. Tappero, and K. Asiedu, "Mycobacterium ulcerans infection," The Lancet, vol. 354, no. 9183, pp. 1013-1018, 1999.

[15] A. Ménard, P. Couppié, D. Sainte-Marie, and R. Pradinaud, "Diagnosis of Mycobacterium ulcerans infection by PCR: about three cases observed in French Guiana," Bulletin de la Societe de Pathologie Exotique, vol. 96, no. 5, pp. 403-405, 2004.

[16] E. Ekaza, A. Kacou-N’Douba, N. C. Oniangué et al., “Contribution of genic amplification in the detection of M. ulcerans in exudates and in cutaneous biopsies in Côte d'Ivoire," Bulletin de la Societe de Pathologie Exotique, vol. 97, no. 2, pp. 95-96, 2004.

[17] http://www.who.int/buruli/laboratory/diagnosis/fr/.

[18] D. Ouattara, G. K. Aka, J. P. Meningaud, A. Sica, L. Kaba, and S. Gadegbeku, "Facial localizations of Buruli ulcers: two cases," Revue de Stomatologie et de Chirurgie Maxillo-Faciale, vol. 104, no. 4, pp. 231-234, 2003.

[19] M. Kondo, I. Kurokawa, Y. Ito, K. I. Yamanaka, T. Yamazaki, and H. Mizutani, "Leg ulcer caused by Mycobacterium ulcerans ssp. shinshuense infection," International Journal of Dermatology, vol. 48, no. 12, pp. 1330-1333, 2009.

[20] K. N'Guessan, P. Guié, P. Iovenitti, G. Carta, V. Loue, and V. Angoi, "Primitive breast localisation of Buruli ulcer in an endemic zone: a rare case," Clinical and Experimental Obstetrics \& Gynecology, vol. 36, no. 4, pp. 265-267, 2009.

[21] E. J. Ecra, I. P. Gbery, B. R. Aka, A. Sangaré, K. kouamé, and L. Dion, "Ulcère de Buruli: à propos de deux cas thoracoabdominaux associés à une pleurésie," Médecine d'Afrique Noire, vol. 48, no. 5, pp. 213-216, 2001. 


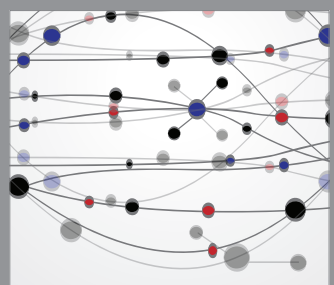

The Scientific World Journal
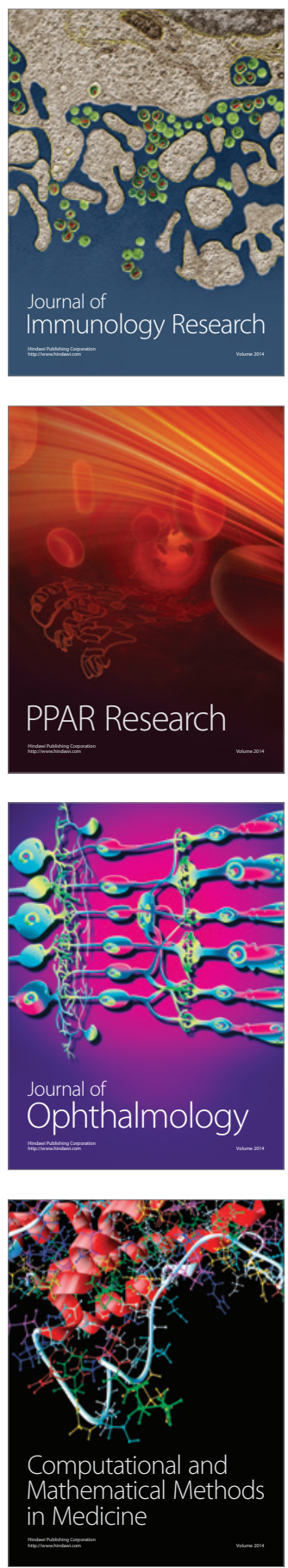

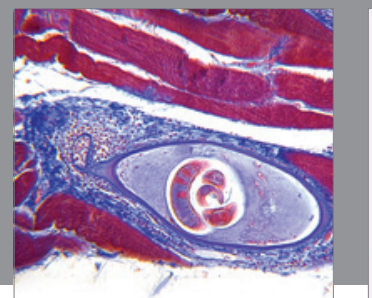

Gastroenterology

Research and Practice
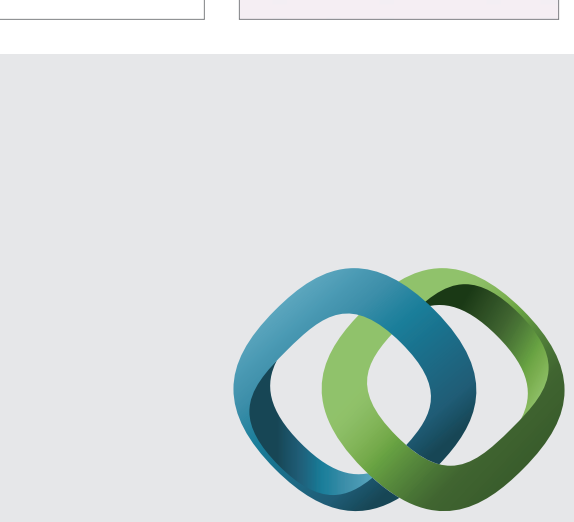

\section{Hindawi}

Submit your manuscripts at

http://www.hindawi.com
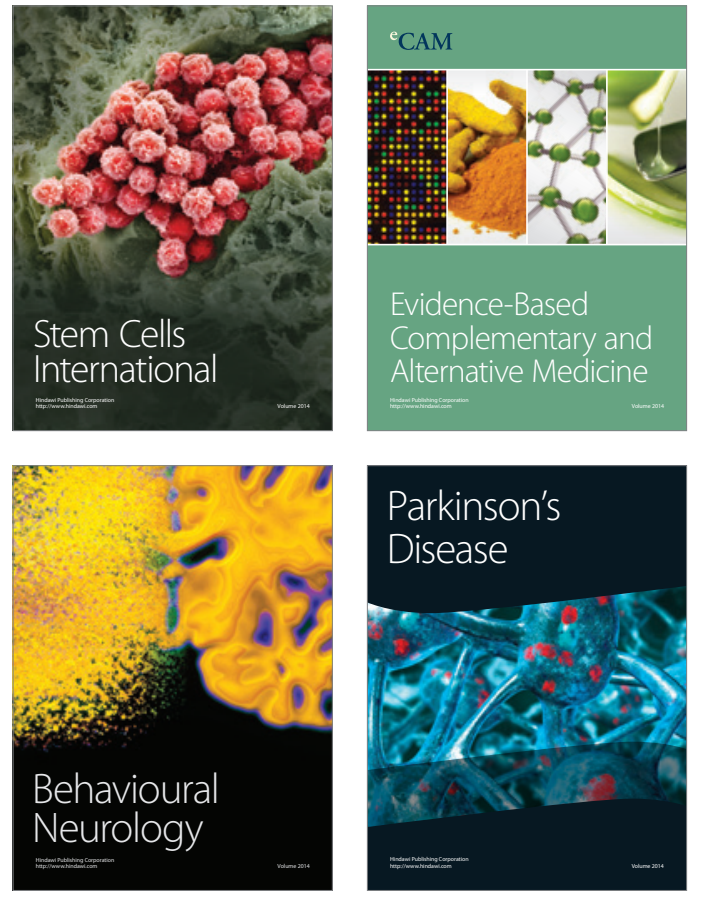
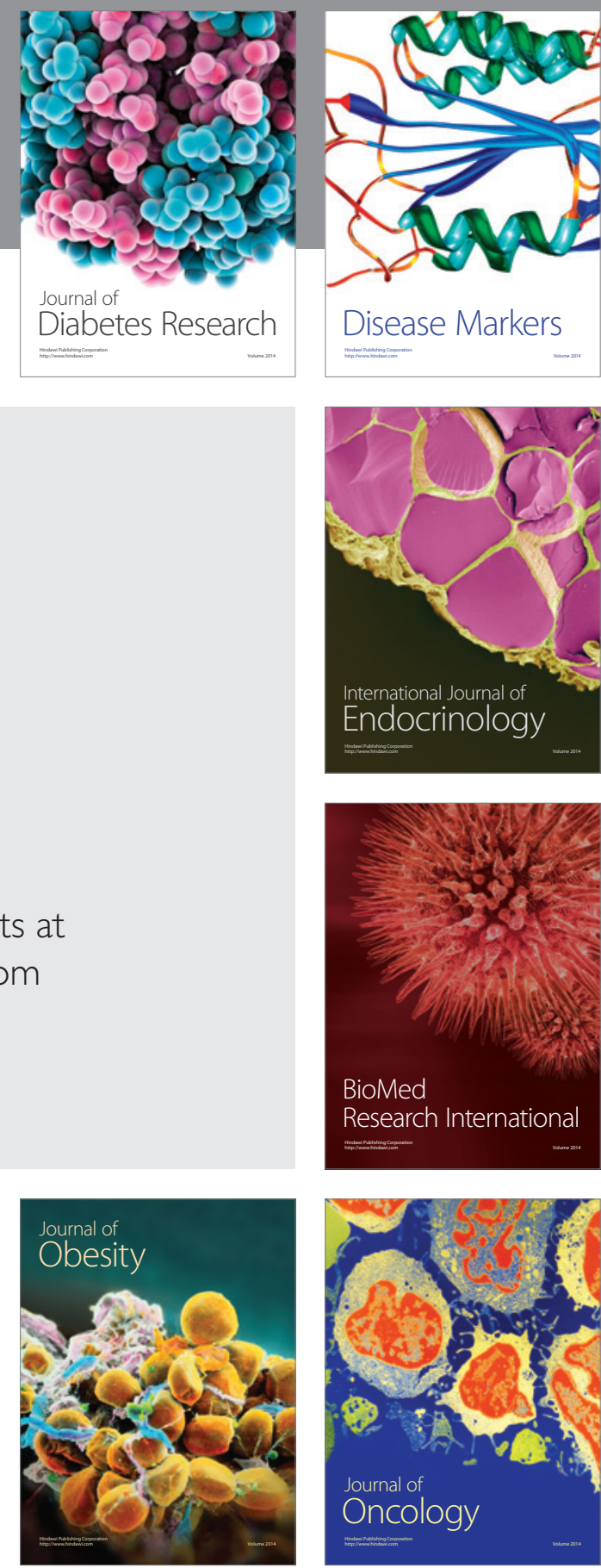

Disease Markers
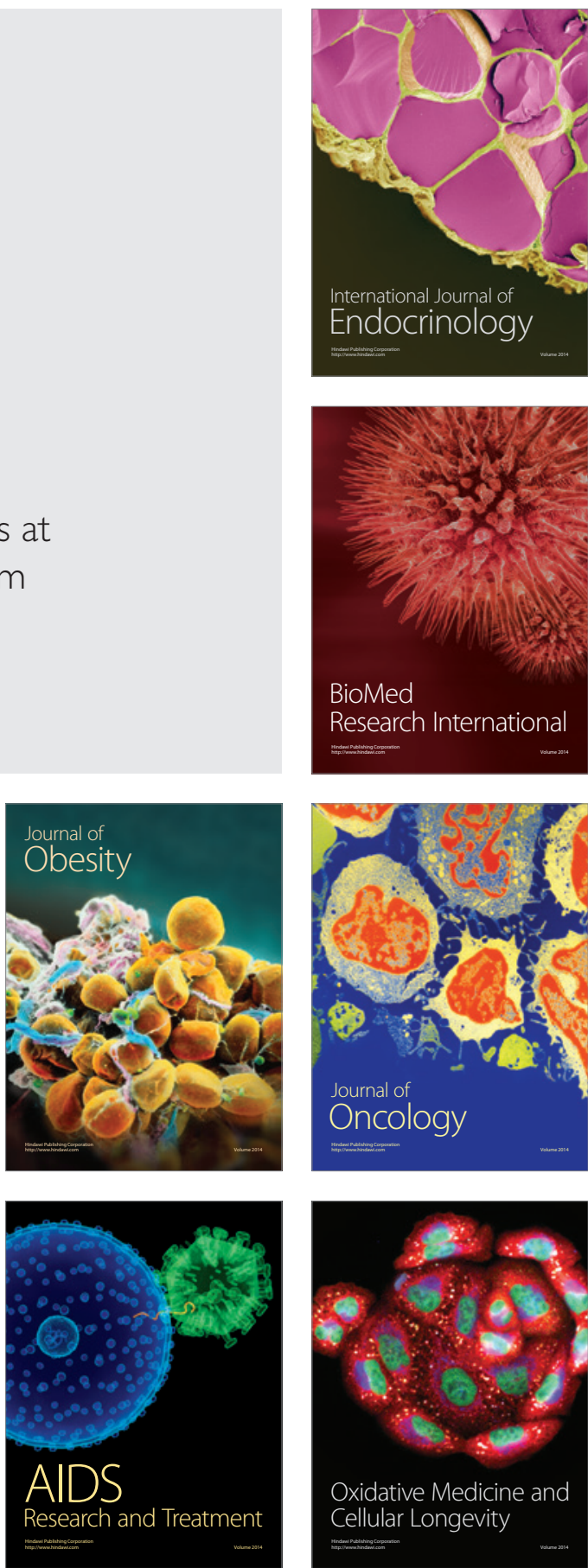